\title{
The Charles Sturt University Remote Telescope Project: Astronomy for Primary School Students
}

\author{
David H. McKinnon and Andrew Mainwaring \\ School of Teacher Education, Charles Sturt University, \\ Bathurst, NSW 2795, Australia \\ dmckinnon@csu.edu.au \\ Received 1999 October 1, accepted 2000 April 1
}

\begin{abstract}
The Charles Sturt University Remote Telescope Project aims to make available to primary school students and their teachers a simple-to-use telescope and CCD camera set up over the Internet. Access to the telescope is supported by a 10 week curriculum unit of Astronomy activities. The telescope is not a robotic device. It is controllable in real time with images being transmitted to the user also in real time. Visitors to the site are able to view what is happening at the telescope without being able to take control of it. This paper describes the project, the software control system and the related curriculum activities. Discussion centres around how to ignite students' and teachers' interest in science and how projects such as this one may lead to more exciting coverage of important topics in the primary and lower secondary schools.
\end{abstract}

Keywords: astronomy education—instrumentation: remote telescopes

\section{Introduction}

The last five years have seen a number of robotic and remote telescope facilities come on-line and which are accessible over the Internet or through modems. One of these, the Bradford Robotic Telescope (http:// www.telescope.org/rti/), is a completely autonomous system that has been available since 1993. Other projects, the most notable of which is Telescopes in Education (http://tie.jpl.nasa.gov/tie), provide remote access in real time via a modem to research grade instruments around the world. Students can access these instruments using The Remote Astronomy Software marketed by the Software Bisque (http://bisque.com).

The Bradford Robotic Telescope's totally autonomous software was developed at the University of Bradford by Dr John Barusch, Mark Cox and others. The system allows users to open an account, receive an observer priority rating that, once obtained, enables the user to submit observation requests on-line. The software schedules the job and the observer receives an email telling them where to locate the images when they have been taken. Professor John Brown, Astronomer Royal for Scotland, has reported Dr Barusch as saying that if they had 365 clear nights a year then they had enough observation requests for the next 84 years (Brown 1998)! Clearly the popularity of being able to take an image 'personally' through the Bradford Telescope is appealing to very many people. Recently, the group has set up a 'studentship program' for university students to undertake research at Bradford. The University also runs a Schools Astronomy Programme targeting students in years 7-12.

The Telescopes in Education (TIE) Project uses a different approach to the Bradford group. Here, schools have to purchase the Remote Astronomy Software (RAS) program suite from the Software Bisque containing The Sky planetarium software and CCDSoft, a windows programme for controlling Apogee and SBIG CCD cameras. Access to the telescopes using the RAS is made via a modem link and the telescope and CCD cameras controlled in real time by the students. The educational materials developed for the TIE project cover all primary and secondary classes from kindergarten to year 12 .

It is against these two large projects, and TIE is very large, that the Charles Sturt University Remote Telescope Project is framed. The project takes elements from both those described above. Specifically, access to the telescope is achieved through the Internet, rather than through a modem link direct to the telescope somewhat like the Bradford Robotic Telescope. Secondly, the telescope is controlled in real time rather like the TIE project. The purpose of this paper, therefore, is to describe more fully the software and hardware systems, and then introduce the educational materials written to support the project.

The aims of the CSU Remote Telescope Project are to:

(1) make available to students in the upper primary school (grades 5 and 6) a telescope that they can control;

(2) provide primary school teachers with good educational materials targeted at their experience level;

(3) motivate students and teachers alike by aiming for the 'Gee, we did that! Isn't science great' impact on students' attitudes; and

(4) build a vehicle for obtaining data on primary students' thinking about, and understanding of, the Universe. 


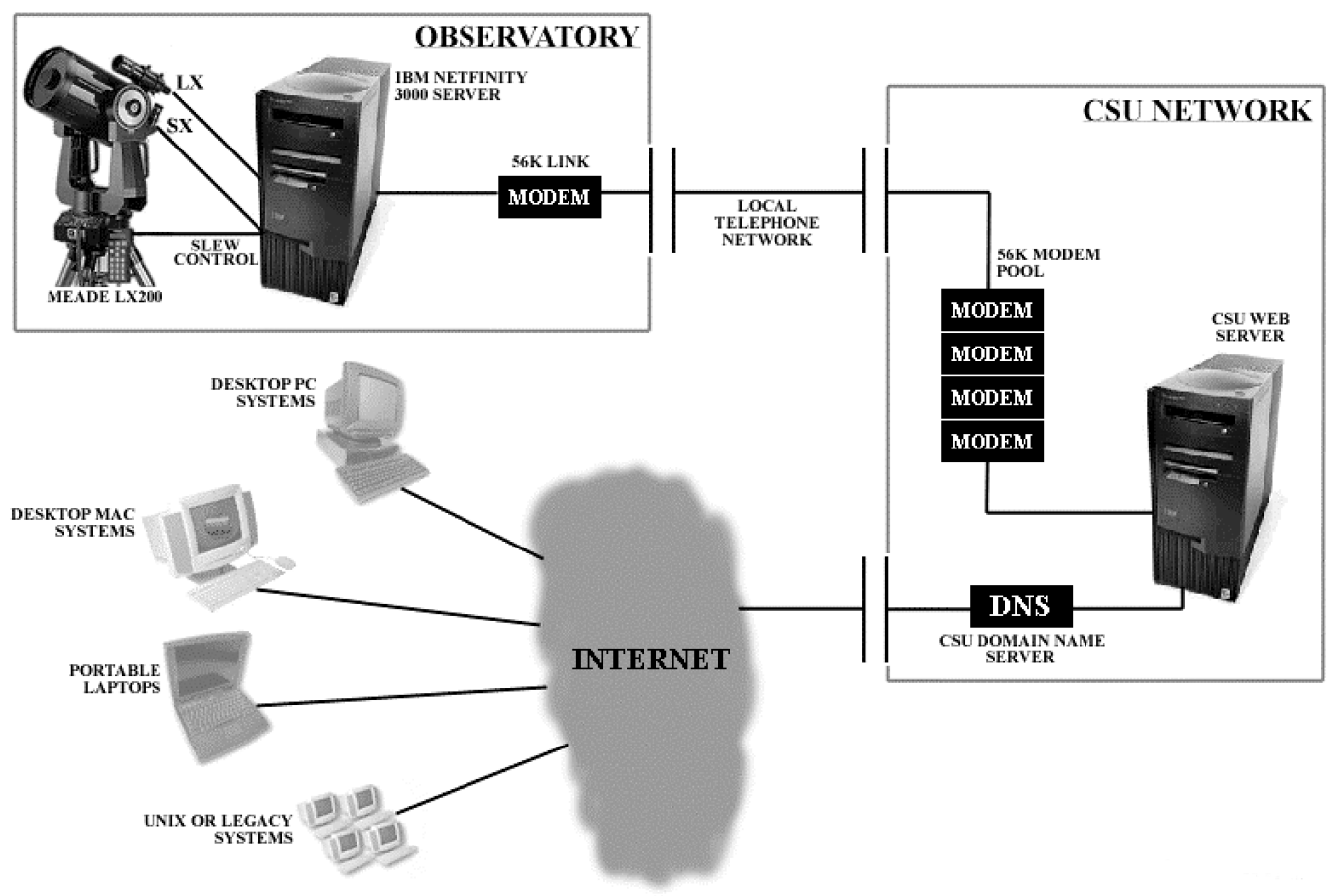

Figure 1-Hardware set-up and communication link to Charles Sturt University.

\section{The Telescope, CCD Cameras and Hardware Set-up}

The telescope is a commercially available 12 inch Meade LX200 that is mounted equatorially. The telescope comes complete with object databases and software that can be used to direct it autonomously to objects in the sky. The telescope has been fitted with an f/6.3 focal reducer. Two CCD cameras are mounted on the telescope: one at the prime focus to give magnified images of the sky, and one piggy backed on the 12 inch with a $135 \mathrm{~mm}$ telephoto lens to give wide angle views of the same region. The CCD cameras are produced by Starlight Xpress (http://www.starlight-xpress.co.uk). Both cameras are interline transfer devices. The prime focus camera is an SX version with $512 \times 256$ pixels each of $12.4 \times 16.6 \mu \mathrm{m}$ on a $6.4 \mathrm{~mm} \times 4.6 \mathrm{~mm}$ chip, and the wide-angle camera is an MX5 version with $500 \times$ 290 pixels each of $9.8 \times 12.6 \mu \mathrm{m}$ on a $4.9 \mathrm{~mm} \times 3.6 \mathrm{~mm}$ chip.

The observatory housing the telescope and other hardware is located near Charles Sturt University, Bathurst, in the central west of New South Wales. The observatory is custom built with a RORO (roll-on-rolloff) roof. The building is a modified 2.3 metre square garden shed with metal louvres fitted to the North, South and East sides and to the roof. This arrangement maintains the internal temperature at no more than one degree Celsius above the ambient air temperature, aided by a thermostatically controlled exhaust fan.

The telescope and CCD cameras are driven by a Netfinity 3000 file server called Black-Hole linked to Charles Sturt University by a high speed modem link running at 56 kilobaud. Black-Hole has its own IP address on the University network. The file server is also networked to a local work station at the first author's home by an Ethernet link running at 100 megabits per second. The telescope and CCD cameras are both controlled by the file server. Figure 1 illustrates the hardware set up and the communication link to the University.

\section{Software System}

The software being used to control access to the telescope and cameras is Windows NT Terminal Server edition 4.01 and running Citrix Metaframe. Users who access the telescope will need to have the Citrix Thin Client software installed on their computer. This comes supplied with a CD-Rom of software, educational materials and extensive image gallery. The Citrix Metaframe software allows clients to use the Internet to access Black-Hole and run control software as though it were on their own desktop. Indeed, the Citrix Metaframe software produces a local screen image that is exactly like a Windows 95, 98 or NT screen. Citrix Metaframe allows the user to save images taken by the cameras to be displayed on their own computer screen and also to be saved to their own hard disk for later off-line image processing by the Starlight Xpress software. An additional feature of Metaframe allows visitors to the view an active session without exercising control over the software or hardware.

The underlying philosophy for software acquisition and web page development has been 'keep it simple stupid (KISS)'. Our target audience, in many instances, consists of teachers whose students have had more computing and Internet experience than they have. The 
Windows NT Terminal Server Edition software allows us to take away a lot of the installation and administration procedures necessary for accessing the observatory's facilities. Upgrades of software are carried out on the file server and this means that new versions do not have to be distributed physically to users.

All the software needed to locate objects in the sky, slew the telescope, and capture a CCD image reside on the observatory server, Black-Hole. From here, the NT environment and applications are emulated to user workstations. Centralising the applications of upgrades or reinstalls can be completely localised and the results made immediately available to users. Other functions of Windows NT Terminal Server Edition that are significant to our project include:

(1) Integration with Windows NT User Manager for Domains-simplifying administration of user accounts. This allows administrators to set encrypted login details for users, their access rights and connection times.

(2) Remote control administration-allows users with such privileges to manage the Terminal Server remotely.

(3) Minimal application licensing-as only one installation is necessary, only one licence needs to be purchased. This removes considerable costs from the user.

To enhance the features of Terminal Server and its server-based computing architecture, we have employed Citrix Metaframe. This software enhances and adds to the simplicity that our project development encompasses. It allows users to connect from any 16- or 32-bit Windows environment using simple Citrix Client Access Software or via Netscape Navigator and Internet Explorer web browsers. Other features we will be utilising include:

(1) Client Device Licensing-establishes multiple server sessions using only one licence. This allows visitors to view the activities on the server without having control in a session.

(2) Minimised network traffic-by sending only screen updates, keystrokes and mouse clicks. Common, non-changing graphics are cached on the user's workstation cutting transmission times considerably.

(3) Client drive mapping-allows local drives to be accessed from server applications for saving of images. 'Cut and paste' features are also available between local and remote programs.

Web page design also employed the 'KISS' philosophy. The application can be broken up into two specific structures. Firstly, there is a public domain web page residing on the Charles Sturt University web server providing general information, a gallery of images, both software and manual downloads, links and resources, contact information, general weather conditions for
Bathurst, and Quicktime movies of the telescope's operation and technical specifications.

Secondly, from the public web site will be a link to the observatory server and its applications using the Metaframe web browser plug-ins. From here, once authenticated, users will have 'live' control of the telescope and CCD cameras or be able to view an active session without control. This software mix and style of development will perhaps minimise the fear, confusion and reluctance often seen with inexperienced users, especially teachers.

\section{Taking Control}

The educational package is constructed in such a way as to teach the primary age children about what astronomical objects are visible above the telescope at any time. Students make decisions about what objects they will image before going on-line. This is done using the SkyGlobe shareware software. Students and their teacher then construct a bid for access to the telescope for a half-hour period at a time convenient to them. The bid is submitted and they are issued with a time dependent logon-id and password. At the appropriate time, the students access the Internet site at http:// www.csu.edu.au/telescope and come through the gateway to the telescope network. On launching The Sky software they see what is visible at the zenith of the observatory at that time. Software controls that users have no need to access are disabled and students have control of the telescope within the prescribed pointing limits set by The Sky software. In effect, they can direct the telescope to the object they wish to image.

The CCD camera software is started in the same way and the users can issue the necessary commands to take an image. The time that it takes for the image to appear on their screen will depend on the Internet. Tests indicate that this is normally between 15 and 30 seconds after the image exposure is completed. Down-load times from the cameras to Black-Hole are normally less than eight seconds. Images are saved at the local computer for later processing.

Warnings are issued at 5 minutes, 2 minutes and 1 minute before the end of the observing session so that the user can tell the telescope to park and logoff.

\section{The Future}

The system is very new and extensive trials have not yet been completed. Nonetheless, preliminary trials indicate that the system is robust. The CCD cameras are parallel port controlled devices and this has presented us with some problems in exploring the world of virtual device drivers to allow Windows NT to take data in through the port. We may yet decide to employ serial port cameras of the SBIG and Apogee types.

The 56 kilobaud modem link to the University may be replaced in the short term by an unregulated microwave link to the CSU network, thus reducing the up- 
load times considerably. Preliminary investigations suggest that such a link would cost around \$3500 and run at around two megabits per second.

When the observatory is moved out of Bathurst to darker skies, a weather station and control software will be installed to prevent the roof being opened when the sky is cloudy or it is raining. The roof opening system will be a modified roll-a-door assembly available commercially but interfaced to the Netfinity 3000 file server.

\section{Conclusion}

These are exciting times for Astronomy and Science Education where students can control remote devices over the Internet. The impact on students' attitudes towards Astronomy in particular and Science in general will be interesting to monitor. Access to such instruments on the Internet which students use themselves to take pictures is likely to be highly motivating. Making an impact in the science classroom is, therefore, a very real possibility that needs to be monitored and evaluated. When these approaches are contrasted with the more usual 'go to the library and do some research' ones employed by many primary school teachers, they present themselves as interesting and exciting.

\section{Acknowledgments}

The authors wish to thank Mike Rebbechi, Executive Director of the Division of Information Technology at Charles Sturt University for making available a grant of $\$ 5000$ and an IP address; various individuals at Charles Sturt University for their support including Pro-Vice Chancellor Bernie O'Donnell, Judith Parker, Bob Meyenn; Starlight Xpress for donating an MX5 black and white camera, and releasing copyright of their image processing and control software to the Project; IBM for making available the Netfinity 3000 file server at much reduced cost, and to Volante Computers for pursuing the donation; The Donovan Astronomical Trust in Australia for a $\$ 600$ grant to purchase an ST-4 camera; and Zircon Systems, Botany, Sydney for their software advice and support.

\section{References}

Brown, J. C. 1998, personal communication 